\title{
Particle Size-Dependent Antibacterial Activity and Murine Cell Cytotoxicity Induced by Graphene Oxide Nanomaterials
}

\author{
Lin Zhao, ${ }^{1}$ Guangxin Duan, ${ }^{1}$ Zaixing Yang, ${ }^{1}$ Jeffrey K. Weber, ${ }^{2}$ Xu Liu, ${ }^{3}$ Shunyi Lu, \\ Xuanyu Meng, ${ }^{1}$ and JiaYing $\mathrm{Xu}^{1}$ \\ ${ }^{1}$ Institute of Quantitative Biology and Medicine, SRMP and RAD-X, Collaborative Innovation Center of Radiation Medicine of \\ Jiangsu Higher Education Institutions, Soochow University, Suzhou 215123, China \\ ${ }^{2}$ IBM Thomas J. Watson Research Center, Yorktown Heights, NY 10598, USA \\ ${ }^{3}$ College of Chemistry, Chemical Engineering and Materials Science, Soochow University, Suzhou 215123, China
}

Correspondence should be addressed to JiaYing Xu; xujiaying@suda.edu.cn

Received 31 March 2016; Accepted 11 May 2016

Academic Editor: Shiren Wang

Copyright (C) 2016 Lin Zhao et al. This is an open access article distributed under the Creative Commons Attribution License, which permits unrestricted use, distribution, and reproduction in any medium, provided the original work is properly cited.

\begin{abstract}
Recent studies have indicated that graphene and its derivative graphene oxide (GO) engage in a wide range of antibacterial activities with limited toxicity to human cells. Here, we systematically evaluate the dependence of GO toxicity on the size of the nanoparticles used in treatments: we compare the cytotoxic effects of graphene quantum dots (GQDs, $<15 \mathrm{~nm}$ ), small GOs (SGOs, 50-200 nm), and large GOs (LGOs, $0.5-3 \mu \mathrm{m}$ ). We synthesize the results of bacterial colony count assays and SEM-based observations of morphological changes to assess the antibacterial properties that these GOs bring into effect against E. coli. We also use Live/Dead assays and morphological analysis to investigate changes to mammalian (Murine macrophage-like Raw 264.7) cells induced by the presence of the various GO particle types. Our results demonstrate that LGOs, SGOs, and GQDs possess antibacterial activities and cause mammalian cell cytotoxicity at descending levels of potency. Placing our observations in the context of previous simulation results, we suggest that both the lateral size and surface area of GO particles contribute to cytotoxic effects. We hope that the size dependence elucidated here provides a useful schematic for tuning GO-cell interactions in biomedical applications.
\end{abstract}

\section{Introduction}

Carbon-based nanomaterials (CBNs) exhibit a congruent combination of chemical and physical properties that make them promising candidate materials for biomedical application [1]. Carbon nanotubes (CNTs) have been explored in a wide array of diagnostic and therapeutic contexts, showing promise in areas like drug delivery, biomedical imaging, and biosensing [2]. The planar analogue to CNTs, graphene, has also inspired an enormous amount of investigation in the fields of nanomedicine and nanotechnology [3]. Graphene's unparalleled thermal and electrical conductivity, remarkable optical properties, high mechanical strength, planar surface, and large accessible surface area make it broadly applicable in biomedical settings $[4,5]$. Graphene, graphene oxide, or graphene-based materials have been extensively explored as either active agents or drug delivery systems [6] in anticancer
[7], antimicrobial [8], and anti-Alzheimer's disease [9] therapies. However, these intriguing applications of graphenebased nanomedicine have been checked by secondary toxicity caused by poor biocompatibility, high agglomeration propensities, and a lack of selectivity toward targeting diseased cells [10].

Graphene oxide (GO), a graphene derivative characterized by hydroxyl, epoxy, and carboxyl functional groups dispersed across its surface, has been shown to be more water-soluble and biocompatible than grapheme [11]. Recent studies suggest that both GO and reduced graphene oxide (rGO, a GO analogue that features fewer surface defects) exert antibacterial effects against $E$. coli [11] and $P$. aeruginosa [12] by inducing oxidative stress and DNA fragmentation. Liu et al. have proposed that the lateral dimension of GO sheets is one of the most relevant properties for bactericidal efficacy: larger (i.e., wider) GO sheets seem to inflict more 
extensive damage upon contact with bacterial cells [13]. A previous computational study (based on molecular dynamics (MD) simulations) probing the underlying interaction mechanisms between $\mathrm{GO}$ and $E$. coli cell membranes also suggests that larger GO nanosheets should more effectively disrupt membrane bilayers via direct cutting/insertion mechanisms and the violent extraction of phospholipid molecules [14]. Data throughout the literature indicate that GO exhibits varying degrees of in vitro cytotoxicity and genotoxicity; $\mathrm{GO}$ is thought to mediate toxicity through the induction of oxidative stress and DNA and cell membrane damage $[5,15$, 16]. Additionally, Li et al. pointed out that GO induced mammalian cell (including J774 murine macrophages, human lung epithelial cells, and human primary keratinocytes) toxicity by disrupting the cytoskeletal organization after it enters cells [17]. Wan et al. proposed that GO could induce autophagosome accumulation and lysosome impairment in primary murine peritoneal macrophages, which might be the source of GO's cytotoxicity [18]. Interestingly, another study concludes that the genotoxicity of GO is inversely correlated with particle size: while micrometer-sized GO becomes moderately genotoxic at higher concentrations, nanometer-sized GO exhibits saturated levels of genotoxicity at all concentrations investigated [19]. Trends in cytotoxicity measured in the same study, however, vary among the cell types tested: both sizes of GO sheets are modestly toxic to A549 cells, the adenocarcinomic human alveolar basal epithelial cells but improve viability in two other cell lines [19]. The reasons behind these apparent discrepancies in cytotoxicity/viability remain to be elucidated; as the above discussion suggests, the effects of GO-based nanomaterials on the well-being of mammalian cells are various, complex, and, at present, not fully understood.

Graphene quantum dots (GQDs) have also been documented to possess optical and electronic properties well suited for biomedical engineering applications [20]. It has been reported that GQDs can effectively inhibit the growth of pathogenic bacteria by generating reactive oxygen species upon photoexcitation [21]. However, though such particles share many physical and chemical properties with GOs, GQDs demonstrated no appreciable toxicity in one mouse model [22].

We here attempt to shed light on a simple question related to GO-cell interactions: how does the size of a GO-based nanomaterial impact its cytotoxicity in specific systems? To systematically study the dependence of GO-induced antimicrobial and mammalian cytotoxicity on particle size, we report the effects of graphene quantum dots (GQDs), small graphene oxide nanosheets (SGOs), and large graphene oxide nanosheets (LGOs) on E. coli and murine macrophage-like cells. The results of this comparative study are explicated in detail below.

\section{Materials and Methods}

2.1. Materials. GQDs $(<15 \mathrm{~nm})$, SGOs $(50-200 \mathrm{~nm})$, and LGOs $(0.5-3 \mu \mathrm{m})$ were purchased from the Chengdu Organic Chemical Co., Chinese Academy of Sciences (Chengdu,
China). The Live/Dead viability kit was purchased from Invitrogen Life Technologies (Carlsbad, CA, USA).

2.2. Characterization of GQDs, SGOs, and LGOs. All samples (GQDs, SGOs, and LGOs) were prepared at a concentration of $10 \mu \mathrm{g} / \mathrm{mL}$ with isotonic and sterile water. Samples were then deposited via spin coating onto a mica substrate and imaged (after a drying period) using atomic force microscopy (AFM, Dimension Icon).

2.3. Bacterial Culture. Cells from E. coli strain $\mathrm{DH} 5 \alpha$ were obtained from the Beyotime Institute of Biotechnology (Jiangsu, China); cells from E. coli strain ATCC-25922 were obtained from the American Type Culture Collection (ATCC, Rockville, MD, USA). Each bacterial strain was incubated in LB (Luria-Bertani) medium overnight to promote an exponential growth phase. Bacterial solutions were then centrifuged $(6000 \mathrm{rpm}, 10 \mathrm{~min})$ and washed twice with sterile saline to remove residual macromolecules and growth medium constituents. Each suspension was diluted with saline solution to $10^{7} \mathrm{CFU} / \mathrm{mL}$ for the experiments that followed.

2.4. Bacterial Viability Test. GQDs, SGOs, and LGOs were freshly prepared with isotonic and sterile saline to achieve uniform dispersion. E. coli cells were incubated in the presence or absence of 20,50, and $100 \mu \mathrm{g} / \mathrm{mL}$ GQDs, SGOs, and LGOs at $37^{\circ} \mathrm{C}$ and a $250 \mathrm{rpm}$ shaking speed for $2 \mathrm{~h}$. Then, colony count assays were applied to test the viability of the bacteria in question. In brief, the treated bacterial dispersions were diluted across four decades in concentration, and $100 \mu \mathrm{L}$ aliquots taken from each group were spread onto LB plates (LB medium with $1.5 \%$ agar). After incubation for $24 \mathrm{~h}$, the colonies on the plates were counted and compared with the control group. The untreated control was considered to be $100 \%$ viable.

2.5. SEM Morphology Investigation of E. coli. To visualize the morphological changes in E. coli after GQD, SGO, and LGO treatment, E. coli (ATCC-25922) at an initial concentration of $10^{7} \mathrm{CFU} / \mathrm{mL}$ were cultured in saline solution containing GQDs, SGOs, or LGOs $(50 \mu \mathrm{g} / \mathrm{mL}, 2 \mathrm{~h})$ and subjected to the vigorous shaking conditions described above (Section 2.4). After centrifugation at $6000 \mathrm{rpm}$ for $10 \mathrm{~min}$, the bacteria were collected and then fixed in a glutaraldehyde solution $(2.5 \%$ glutaraldehyde in $0.2 \mathrm{M}$ sodium cacodylate/hydrochloric acid buffer, $\mathrm{pH} 7.5$ ) at $4^{\circ} \mathrm{C}$ for $2 \mathrm{~h}$. After repeated centrifugation with double-distilled water $(6000 \mathrm{rpm}, 10 \mathrm{~min})$, E. coli cells were dehydrated in a graded series of ethanol solutions (25, $50,75,85,95,100$, and $100 \mathrm{v} / \mathrm{v} \%$ ) for $10 \mathrm{~min}$ each. Lastly, all the samples were dried to remove ethanol and mounted onto an aluminum stub, coated by gold sputter, and examined under a scanning electron microscope (S-4700, Japan).

2.6. Murine Cell Cultures. Murine macrophage-like Raw 264.7 cells were obtained from the American Type Culture Collection (ATCC) (Rockville, Maryland, USA). Cells were cultured in Dulbecco's modified Eagle's medium (Gibco, 
Shanghai, China), supplemented with $10 \%$ fetal calf serum, Lglutamine $(5 \mathrm{mmol} / \mathrm{L})$, nonessential amino acids $(5 \mathrm{mmol} / \mathrm{L})$, penicillin $(100 \mathrm{U} / \mathrm{mL})$, and streptomycin $(100 \mathrm{U} / \mathrm{mL})$ (Invitrogen) at $37^{\circ} \mathrm{C}$ in a humidified $5 \% \mathrm{CO}_{2}$ atmosphere.

2.7. Cell Morphology and Viability Assay of Raw 264.7 Cells. Raw 264.7 cells were seeded in 24 -well plates at a density of $2 \times 10^{4}$ per well and incubated in complete culture medium containing $10 \%$ FBS. After cultured overnight, Raw 264.7 cells reached $\sim 60 \%$ confluence. Then, the cells were treated for another $24 \mathrm{~h}$ by nanoparticles dispersions, freshly prepared by diluting GQDs, SGOs, and LGOs stock solution in serum-free DMEM medium at the concentration of 5$50 \mu \mathrm{g} / \mathrm{mL}$. Cell cultured without nanoparticles were taken as the negative control. Subsequently, after extensive washing with PBS, cell morphologies were studied using visible light microscopy. At the same time, cell viability was tested using a Live/Dead assay. Briefly, $1 \mathrm{~mL}$ of PBS containing $4 \mu \mathrm{L}$ of $2 \mathrm{mM}$ ethidium homodimer-1 (EthD-1) assay solution and $2 \mu \mathrm{L}$ of $50 \mu \mathrm{M}$ calcein AM assay solution was prepared. $200 \mu \mathrm{L}$ of the Live/Dead solution was added to each well for $15 \mathrm{~min}$ in an incubator at $37^{\circ} \mathrm{C}$. The staining solution was removed and the samples were then imaged under a fluorescence microscope (Olympics IX73) with $494 \mathrm{~nm}$ (green, calcein) and $528 \mathrm{~nm}$ (red, EthD-1) excitation filters. Live and dead cells were also counted, and cell proliferation was expressed as the percent cell viability. Untreated cells were considered to be $100 \%$ viable.

2.8. Statistical Analysis. Data are expressed as means \pm standard deviations collected from at least two independent experiments. Statistical comparisons between groups were carried out using a two-tailed Student's $t$-test. All statistical tests were conducted using SPSS version 17.0 (IBM Co., Shanghai, China). Differences were considered statistically significant when the corresponding $P$ values were 0.05 or less.

\section{Results and Discussion}

3.1. Physical Properties of GQD, SGO, and LGO. Prior to exploring the cytotoxic effects of GO nanoparticles, we first sought to characterize the nanomaterials of interest in isolation. AFM images (Figures $1(\mathrm{a})-1(\mathrm{c})$ ) indicate that the three types of GO sheets (GQDs, SGOs, and LGOs) range in thickness from 0.8 to $1.5 \mathrm{~nm}$. Consistent with reports in the literature, single layer GO sheets exhibited a uniform thickness of about $1 \mathrm{~nm}$; GO sheets are expected to be thicker than pristine graphene sheets due to the presence of oxygen-containing functional groups and sp3-hybridized carbon atoms on the oxidized surface $[23,24]$. However, it was previously reported that single-layered GQDs had an average thickness of about $0.4 \mathrm{~nm}$ [25], suggesting that the GQDs used in the present study are composed of 2-3 graphene layers. Contrast among the lateral dimensions of the materials used in our experiments was nonetheless high (Figures 1(a)1(c)): GQDs were comparatively small $(<15 \mathrm{~nm})$, SGOs were intermediate in size (about 50-200 nm), and LGOs (about $0.5-3 \mu \mathrm{m}$ ) were the largest of the materials studied. In theory, functional GQDs need to maintain a uniform dimension of $3-5 \mathrm{~nm}[25,26]$. Practically, ready aggregation likely caused the GQDs in this work to assume a larger range of sizes.

3.2. Antibacterial Activity of GQD, SGO, and LGO. To determine the antibacterial properties of GQDs, SGOs, and LGOs, a simple colony count method was employed. As illuminates (Figure 2), 20, 50, and $100 \mu \mathrm{g} / \mathrm{mL}$ GQDs had no significant antibacterial effects on the two $E$. coli strains studied $(P>$ $0.05)$. However, SGOs and LGOs were both observed to significantly diminish the survival rate of bacteria. Furthermore, LGOs proved to exhibit stronger bactericidal effects than did SGOs at comparable concentrations: $\mathrm{DH} 5 \alpha$ cell viability, for instance, was decreased to $(29.77 \pm 3.59) \%$ and $(5.32 \pm 2.50) \%$ after treatment with $50 \mu \mathrm{g} / \mathrm{mL}$ SGOs and LGOs, respectively $(t=9.68, P<0.01)$. A similar disparity was observed in cells from the ATCC-25922 strain. Together, our results indicate that SGO and LGO can both inhibit bacterial growth, with LGO demonstrating the stronger effect. Previously, Liu et al. established that graphene oxide (GO) had more outstanding antibacterial properties than reduced graphene oxide $(\mathrm{rGO})$, graphite (Gt), and graphite oxide (GtO), perhaps due to its smallest average size among these materials [27]. However, the authors subsequently point out that GO sheets with larger dimensions had stronger bactericidal effects [13]. It has been proposed that larger GO sheets could cover the most of bacterial cell surface, while smaller size GO probably aggregated on local bacterial surface. Once cells were covered by larger size GO, the proliferation should be inhibited, and necessary nutrients could be blocked outside [13]. In contradistinction to this evidence, some data suggest that GO can actually exhibit favorable biocompatibility and promote the proliferation of bacteria through the maintenance of cell membrane integrity [28]. Additionally, GO aggregate supplements to LB medium have been shown to enhance bacterial growth [29]. In recent molecular dynamics simulations, we found that graphene could adsorb various serum proteins to a point of near-complete surface coverage; aromatic and electropositive protein residues seemed to play a particularly important role in the adsorptive process [data under review]. One might speculate that the adsorption of nutrients from LB medium (e.g., peptone) onto GO particles explains their transition from bactericide to the promotion of bacterial growth. Notably, bacteria were cultured in the absence of protein supplements after the addition of GO particles in our experiments. Interestingly, GOs did not cause equivalent amounts of damage to the two strains of $E$. coli studied: only $(5.32 \pm 2.50) \%$ DH $5 \alpha$ cells could survive at a $50 \mu \mathrm{g} / \mathrm{mL}$ concentration of LGOs, but (15.31 \pm 7.62$) \%$ ATCC- 25922 cells escaped death under the same conditions.

3.3. SEM Images of E. coli after Incubation with GQDs, $S G O s$, and LGOs. In light of the deleterious effects that SGOs and LGOs were shown to act on E. coli viabilities, we used scanning electron microscopy (SEM) to examine the morphological changes that occur in bacterial cells upon GO treatment. Bacteria in the untreated group remained rod-like in shape and exhibit smooth membrane surfaces in images 


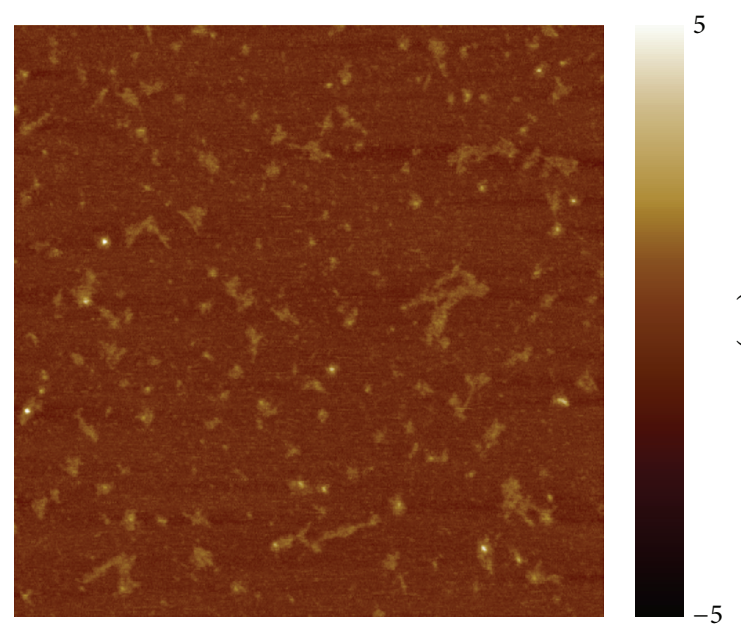

(a)

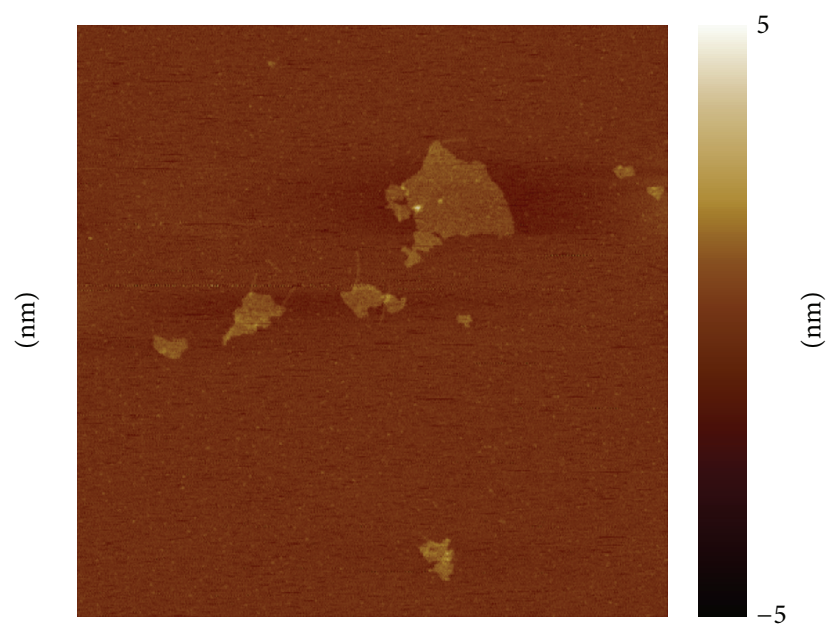

(b)

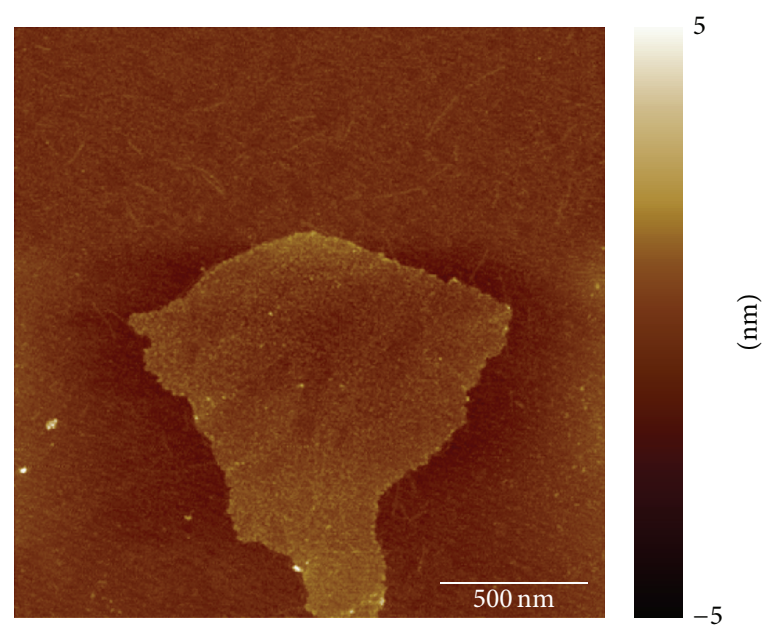

(c)

FIGURE 1: Characterization of GQDs, SGOs, and LGOs. AFM images of GQD (a), SGO (b), and LGO (c) sheets at a concentration of $10 \mu \mathrm{g} / \mathrm{mL}$ on freshly cleaved mica surfaces.

(Figure 3(a)). No significant changes in morphology are seen within the GQD $(50 \mu \mathrm{g} / \mathrm{mL}, 2 \mathrm{~h})$ group; the integrity and smoothness of the associated bacterial membranes remain intact (Figure 3(b)). However, after two hours of exposure to SGOs and LGOs at same concentration, obvious changes to bacterial shape and properties took place. In the images included in Figures 3(c) and 3(d), membranes appear to be inhomogeneous and SGOs and LGOs are observed to cover the bacterial cells under scrutiny. The E. coli cells seem to adhere and bind to skeletons of aggregated GO sheets; cell bodies are shriveled, twisted, cupped, or otherwise distorted by mechanical forces applied by both SGOs and LGOs (Figures 3(c) and 3(d)). Within the LGO treatment group, some bacterial cells ruptured in violent fashion, visibly dispersing cytoplasm into the extracellular surroundings (Figure 3(d)). Our results thus indicate that SGOs and LGOs cause significant structural damage to bacterial that, presumably, facilitates growth inhibition and cell death. Previously, Chen et al. reported that the toxicity of GO to bacteria could likely be attributed to a collusion of local GO-induced defects in cell membranes which ultimately results in cell lysis [30]. Other studies suggest that modified GOs (e.g., graphene films on $\mathrm{Cu}$ and $\mathrm{Ge}$ [31] and $\mathrm{ZnO} / \mathrm{GO}$ composites [32]) also induce bacterial death via mechanisms involving membrane damage. Furthermore, a previous MD simulation study corroborates the notion that GO nanosheets can both cut/be inserted into bacterial membranes and violently extract the phospholipid molecules from the membrane surface [14]. The present work provides further evidence that GO causes bacterial death by physically damaging the bacterial membrane structure.

3.4. Cytotoxic Effects of GQD, SGO, and LGO. As in bacterial systems, consistent morphology is a good indicator of activity and health in mammalian cells. To probe the cytotoxic effects of GQDs, SGOs, and LGOs on a specific eukaryotic system, we examined Murine macrophage-like Raw 264.7 cells after exposure to GO-based nanomaterials. The cells in question were incubated in $10 \mu \mathrm{g} / \mathrm{mL}$ GQD, SGO, and LGO solutions without FBS for $24 \mathrm{~h}$. Compared to the untreated control, 


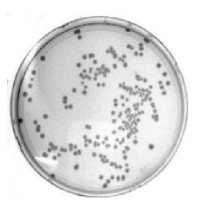

0

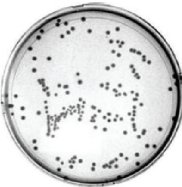

20

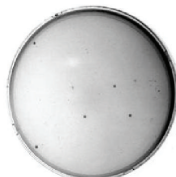

50

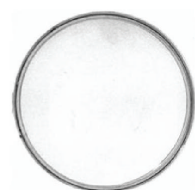

100

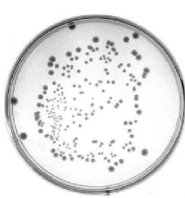

0

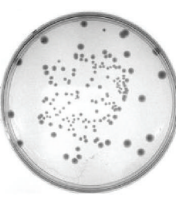

20

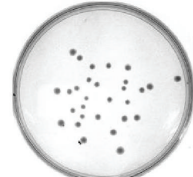

50

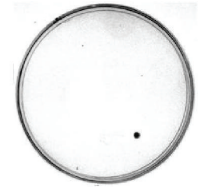

100

(A)

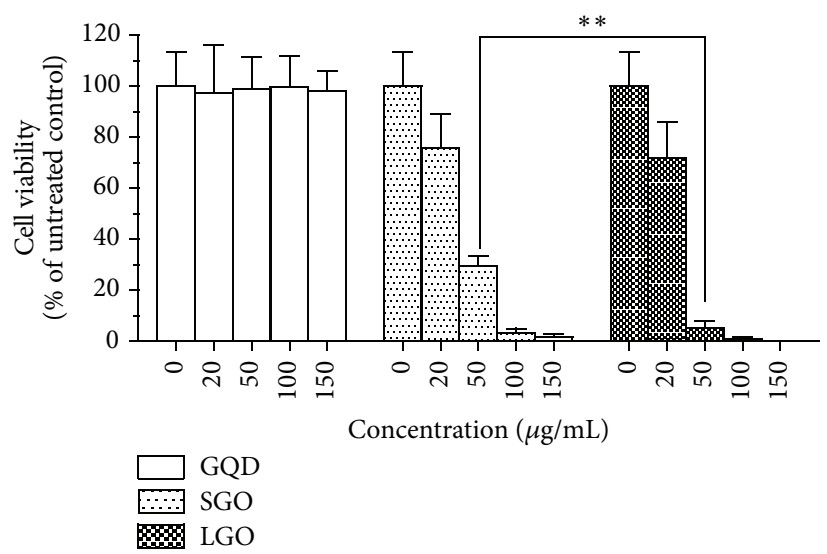

(B)

(a) E. coli $\mathrm{DH} 5 \alpha$

LGO $(\mu \mathrm{g} / \mathrm{mL})$

(A)

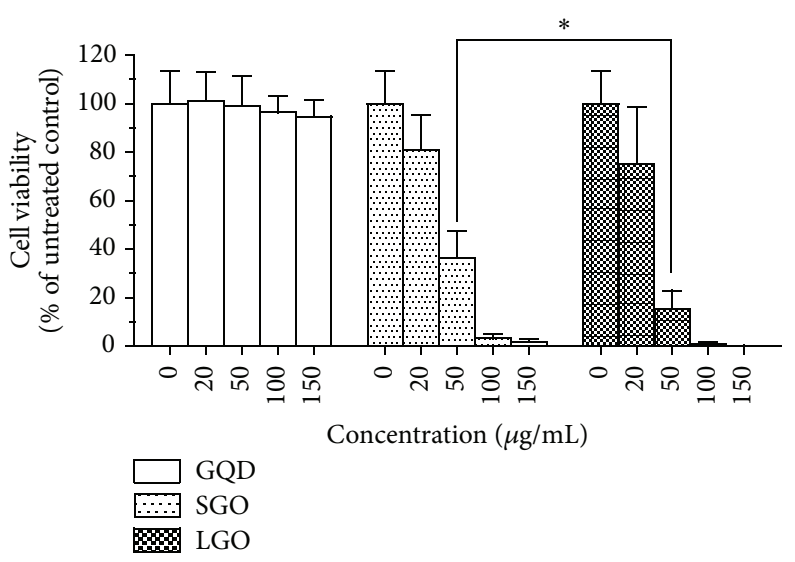

(B)

(b) E. coli ATCC25922

FIGURE 2: Antibacterial activities of GQD, SGO, and LGO. Representative photographs of E. coli ((a) E. coli DH5 $\alpha$; (b) E. coli ATCC-25922) colonies on LB-Agar culture plates after $0-100 \mu \mathrm{g} / \mathrm{mL}$ LGO treatment for $2 \mathrm{~h}$. Our viability assay for E. coli consists of standard plate counting after $0-150 \mu \mathrm{g} / \mathrm{mL}$ GQD, SGO, and LGO treatment for $2 \mathrm{~h}$. The colonies on plates in different groups were compared with an untreated control (considered to be $100 \%$ viable). The data are presented as mean \pm SD values derived from three independent experiments $\left({ }^{*} P<0.05\right.$; $\left.{ }^{* *} P<0.01\right)$.

Raw 264.7 cells in the GQD-treated group appeared slightly swollen; a considerable number of GQD-treated cells became polygonal (Figure 4(a), yellow arrow), possibly indicating internal damage. After an equivalent treatment with SGOs and LGOs, however, cells were observed to be seriously damaged. Most cells in the SGO and LGO groups became inflated and riddled with large cytoplasmic vacuoles, as the red arrows in Figure 4(a). We also found that a number of cells experienced changes in orientation after SGO and LGO treatment. Groups of cells became disordered and only loosely adhered to their substrates; finally, many cells floated in isolation into the medium (indicating that such cells died).

It has been reported that if properly administered and delivered, GO could form the basis for an effective anticancer treatment by activating autophagous pathways that induce cell death $[18,33,34]$. The formation of large vacuoles in the SGO- and LGO-treated groups may be related to the genesis of autolysosomes; more investigation regarding this particular hypothesis is certainly warranted. In principle, our results indicate that the larger GO nanosheets are cytotoxic in the absence of supplemental serum proteins. However, our experiments provide no evidence that such cytotoxic effects are specific to or consistent among various cell types; we suspect that proper targeting and delivery would present a challenge to the development of effective GO-based anticancer therapies. Some data suggest that GO can even enhance the viabilities of certain human cancer cells [19]; we find no such GO-induced increase in viability in the murine macrophage-like cells studied here.

To quantify the survival rates of GO-treated murine cells, we applied fluorescence probes to track live and dead cells in solution. Ethidium homodimer-1 (EthD-1) is able to enter damaged/dead cells and produce a bright red fluorescence signal, while calcein AM is retained by live cells and produces green light. As Figure 4(a) illustrates, no obvious changes in the GQD group were observed as compared to the untreated control. By contrast, the number of cells emitting green light was diminished in the SGO and LGO groups, and the number of cells emitting red light increased, confirming the strong cytotoxic effects of SGOs and LGOs. Based on the viability graph (Figure 4(b)), we also noted that the inhibitory effect of SGOs was slightly weaker than the comparable effect induced by LGOs. Overall, we thus conclude that both SGOs and LGOs are able to induce murine cell death, and the extent of cytotoxic effects descends as LGOs > SGOs > GQDs. Several studies have demonstrated that the toxicity of GO and GO derivative is also closely correlated with levels of oxidative stress in cells $[35,36]$. Further investigation in this 


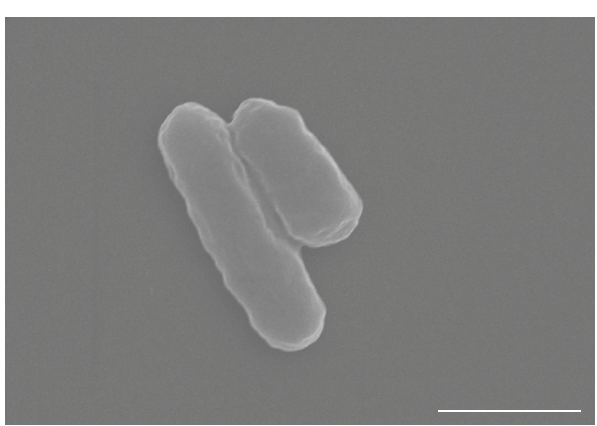

(a)

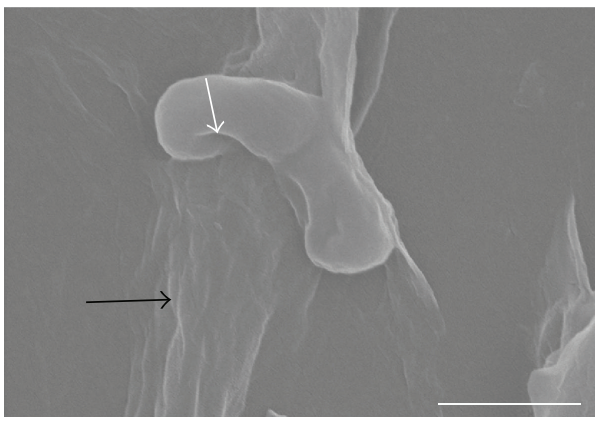

(c)

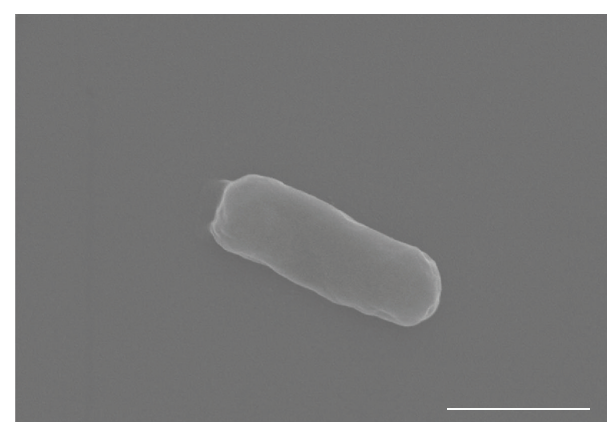

(b)

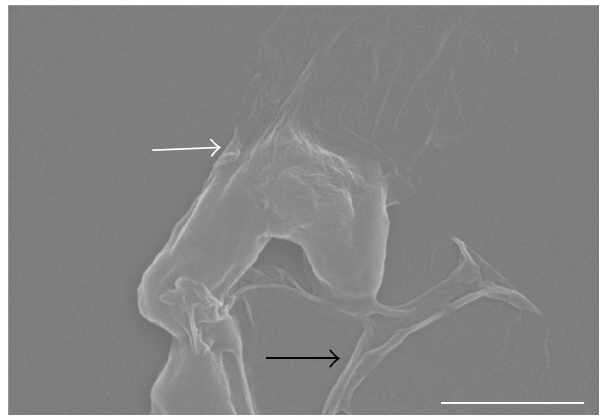

(d)

FIGURE 3: SEM morphology of E. coli cells after GQD, SGO, and LGO treatment. SEM morphology of E. coli cells (strain ATCC-25922): (a) control; (b) E. coli treated with $50 \mu \mathrm{g} / \mathrm{mL}$ GQD solution for $2 \mathrm{~h}$; (c) E. coli treated with $50 \mu \mathrm{g} / \mathrm{mL}$ SGO solution for $2 \mathrm{~h}$; (d) E. coli treated with $50 \mu \mathrm{g} / \mathrm{mL}$ LGOs for $2 \mathrm{~h}$. White arrows: ruptured E. coli cells. Black arrows: GO sheet aggregates. Scale bar $=1 \mu \mathrm{M}$.

area would improve our understanding of GO's systemic mechanism of cytotoxicity and provide essential support for further evaluation of GO's safety in biomedical applications.

\subsection{Rationalization of Size Dependence Based on Previous} Simulation Results. Recently, Tu et al. employed molecular dynamics (MD) simulations to investigate the detailed mechanisms by which graphene nanosheets interact with bacterial cell membranes [14]. As mentioned above, graphene sheets were observed to damage phospholipid bilayers according to two distinct (yet not mutually exclusive) modes of disassembly: those of direct insertion and violent lipid extraction. The destructive implications of direct membrane penetration on the part of graphene are obvious, as the inserted carbon nanosheet can partition groups of lipids and act to cut the bilayer into separate pieces. Under the lipid extraction paradigm, the impinging graphene sheet induces an almost "vapor-like" phase in nearby membrane segments: individual lipid molecules jump from the membrane and climb onto the hydrophobic graphene surface. Reductions in the local lipid density ensue, creating temporary voids in the surface which ultimately strain the entire bilayer. Both mechanisms can act in concert to deform and divide the membrane surface, as lipid extraction creates pockets that accommodate direct insertion [14].

Direct computational results concerning the sizedependence of membrane damage are difficult to obtain, as the range of graphene sizes tractable for simulation is very small. However, one can appeal to simple physical arguments to contextualize the size dependence of graphene's antibacterial properties in terms of the above microscopic mechanisms of interaction. Larger nanosheets should create more extensive cuts in bacterial membranes upon direct penetration in a manner proportional to the linear dimension of the graphene present. Naturally, these longer cuts would create a more damaging matrix of incisions that ultimately translate into heightened cytotoxicity. Furthermore, the physics of lipid extraction should scale (to some extent) with the surface area of the interacting graphene: in simulations, both sides of graphene nanosheets became covered with adsorbed phospholipids [14]. Correspondingly, the capacity for lipid extraction should increase markedly with larger nanosheets. Synthesizing the effects of both mechanisms, it is sensible that antibacterial activity would be positively correlated with graphene particle size. Though one might expect graphene/GO aggregation to also increase in extent as the size of individual nanosheets increases, such effects do not appear to mitigate increased cytotoxicity that comes from larger GO particles in our experiments. It may be interesting to simulate the effects of small graphene/GO aggregates on bacterial membranes in the future.

\section{Conclusion}

We have thus demonstrated that the antibacterial activity and murine macrophage-like cell cytotoxicity mediated by 

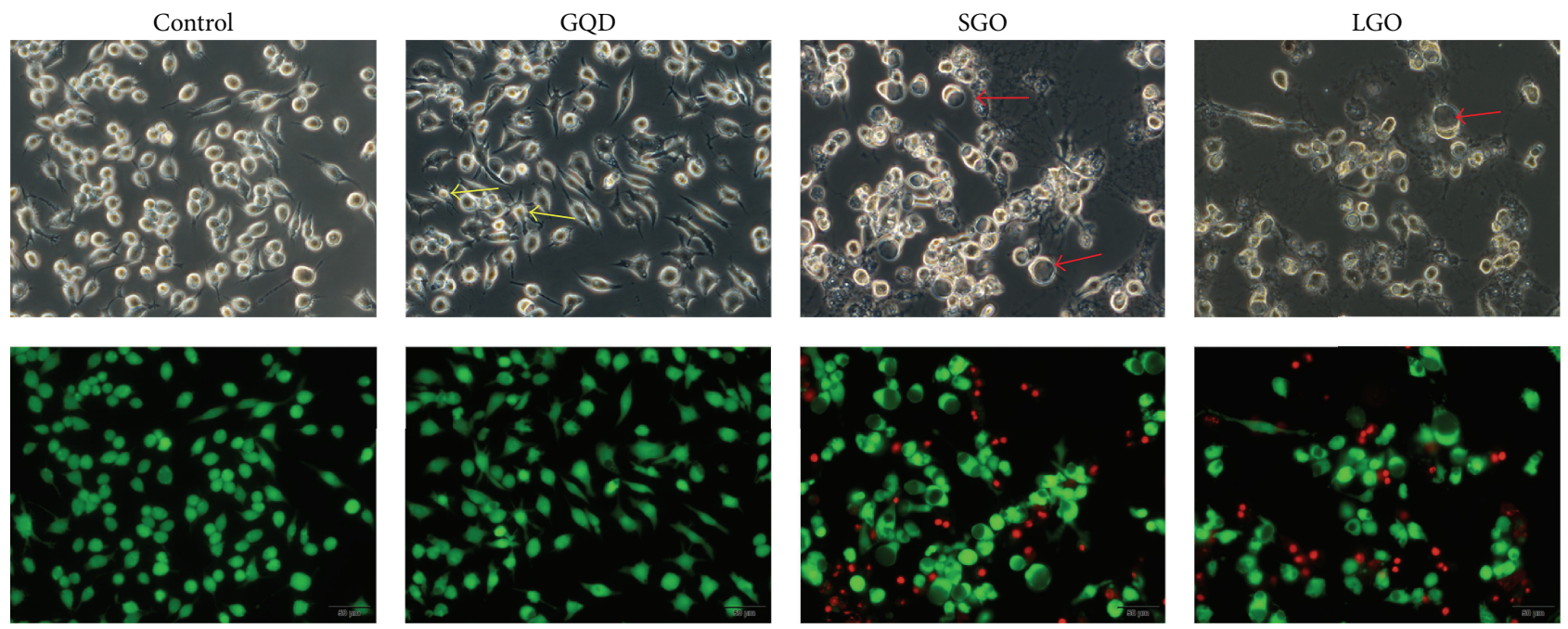

(a)

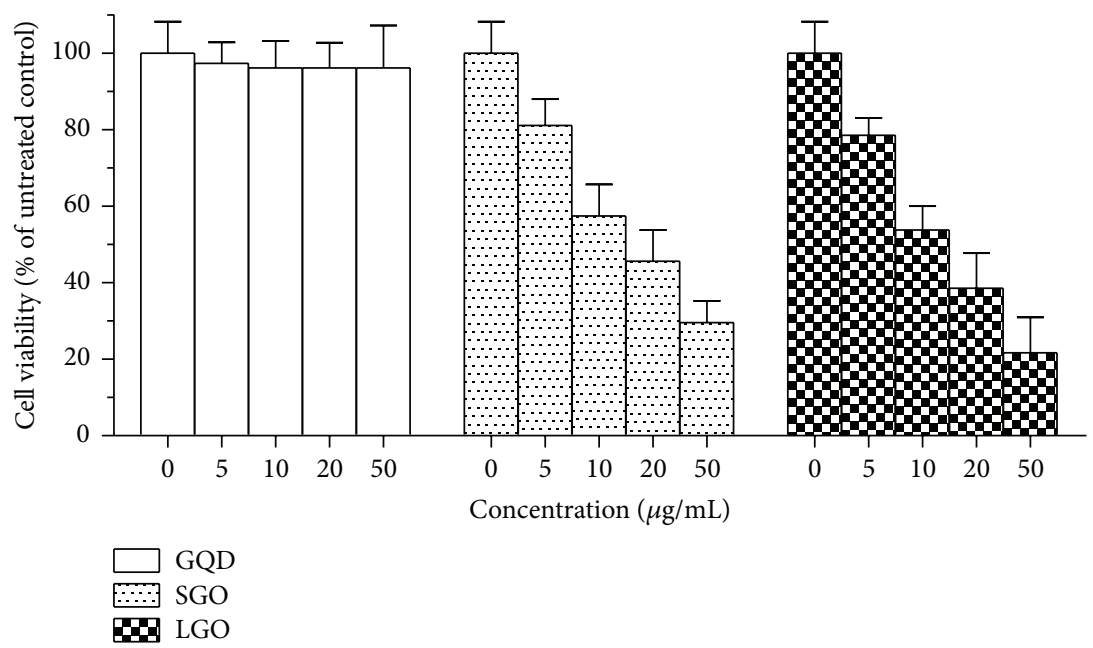

(b)

Figure 4: Cytotoxic effects of GQDs, SGOs, and LGOs. Morphology of Raw 264.7 cells ((a) upper). Raw 264.3 cells were treated with $10 \mu \mathrm{g} / \mathrm{mL}$ solutions of GQDs, SGOs, and LGOs for $24 \mathrm{~h}$. Yellow arrows: polygonal Raw 264.3 cells. Red arrows: Raw 264.3 cells with large vacuoles. The cytotoxicity assay of Raw 264.7 cells consisted of a live (cells illuminated by green light, (a) lower)/dead (cells illuminated by red light, (a) lower) assay after $10 \mu \mathrm{g} / \mathrm{mL}$ GQD, SGO, and LGO treatment for $24 \mathrm{~h}$. The viability assay by CCK-8 of Raw $264.7 \mathrm{cells}$ after 0-50 $\mu \mathrm{g} / \mathrm{mL}$ GQD, SGO, and LGO treatment (without FBS) for $24 \mathrm{~h}$ (b). The treated groups were compared with an untreated control (considered to be $100 \%$ viable). The data are presented as mean \pm SD values derived from three independent experiments.

GO sheets are positively correlated with nanoparticle size. Furthermore, we provide evidence that GO disturbs bacterial structure by twisting and distorting cell bodies; such structural consequences are consistent with the robust mechanical and physical properties of the nanomaterials in question. We have also discussed how the observed size-dependence complements intuition derived from simulations of GO-mediated destruction of bacterial membranes. Overall, we hope our results improve the understanding of how particle size might be used to tune GO-cell interactions and cytotoxicities in both bacterial and mammalian systems, so that GO-based applications might become more tractable throughout an array of biomedical fields.

\section{Competing Interests}

The authors declare that they have no competing interests.

\section{Authors' Contributions}

Lin Zhao and Guangxin Duan contributed equally to this paper.

\section{Acknowledgments}

The authors thank Professor Ruhong Zhou for helpful discussions. This work was supported by the project of Natural Science Foundation of Jiangsu Province (14KJB310018), 
China Postdoctoral Science Foundation of the Fifty-Seventh Batch of Funds (2015M571889), the Priority Academic Program Development of Jiangsu Higher Education Institutions (PAPD), Jiangsu Provincial Key Laboratory of Radiation Medicine and Protection, the Research Innovation Program for College Graduates of Jiangsu Province (KYLX15_1205), and the Suzhou Scientific and Technology Program (SYS201511).

\section{References}

[1] C. Cha, S. R. Shin, N. Annabi, M. R. Dokmeci, and A. Khademhosseini, "Carbon-based nanomaterials: multifunctional materials for biomedical engineering," ACS Nano, vol. 7, no. 4, pp. 2891-2897, 2013.

[2] H. Gong, R. Peng, and Z. Liu, "Carbon nanotubes for biomedical imaging: the recent advances," Advanced Drug Delivery Reviews, vol. 65, no. 15, pp. 1951-1963, 2013.

[3] X. Zhou and F. Liang, "Application of graphene/graphene oxide in biomedicine and biotechnology," Current Medicinal Chemistry, vol. 21, no. 7, pp. 855-869, 2014.

[4] L. Feng and Z. Liu, "Graphene in biomedicine: opportunities and challenges," Nanomedicine, vol. 6, no. 2, pp. 317-324, 2011.

[5] T. Nezakati, B. G. Cousins, and A. M. Seifalian, "Toxicology of chemically modified graphene-based materials for medical application," Archives of Toxicology, vol. 88, no. 11, pp. 1987-2012, 2014.

[6] J. Liu, L. Cui, and D. Losic, "Graphene and graphene oxide as new nanocarriers for drug delivery applications," Acta Biomaterialia, vol. 9, no. 12, pp. 9243-9257, 2013.

[7] D.-J. Lim, M. Sim, L. Oh, K. Lim, and H. Park, "Carbon-based drug delivery carriers for cancer therapy," Archives of Pharmacal Research, vol. 37, no. 1, pp. 43-52, 2014.

[8] G. S. Martynková and M. Valášková, "Antimicrobial nanocomposites based on natural modified materials: a review of carbons and clays," Journal of Nanoscience and Nanotechnology, vol. 14, no. 1, pp. 673-693, 2014.

[9] J. Li, Q. Han, X. Wang et al., "Reduced aggregation and cytotoxicity of amyloid peptides by graphene oxide/gold nanocomposites prepared by pulsed laser ablation in water," Small, vol. 10, no. 21, pp. 4386-4394, 2014.

[10] S. Shi, F. Chen, E. B. Ehlerding, and W. Cai, "Surface engineering of graphene-based nanomaterials for biomedical applications," Bioconjugate Chemistry, vol. 25, no. 9, pp. 1609-1619, 2014.

[11] W. Hu, C. Peng, W. Luo et al., "Graphene-based antibacterial paper," ACS Nano, vol. 4, no. 7, pp. 4317-4323, 2010.

[12] S. Gurunathan, J. W. Han, A. Abdal Dayem, V. Eppakayala, and J.-H. Kim, "Oxidative stress-mediated antibacterial activity of graphene oxide and reduced graphene oxide in Pseudomonas aeruginosa," International Journal of Nanomedicine, vol. 7, pp. 5901-5914, 2012.

[13] S. Liu, M. Hu, T. H. Zeng et al., "Lateral dimension-dependent antibacterial activity of graphene oxide sheets," Langmuir, vol. 28, no. 33, pp. 12364-12372, 2012.

[14] Y. Tu, M. Lv, P. Xiu et al., "Destructive extraction of phospholipids from Escherichia coli membranes by graphene nanosheets," Nature Nanotechnology, vol. 8, no. 8, pp. 594-601, 2013.

[15] Y. Chang, S.-T. Yang, J.-H. Liu et al., "In vitro toxicity evaluation of graphene oxide on A549 cells," Toxicology Letters, vol. 200, no. 3, pp. 201-210, 2011.
[16] L. Agemy, K. N. Sugahara, V. R. Kotamraju et al., "Nanoparticleinduced vascular blockade in human prostate cancer," Blood, vol. 116, no. 15, pp. 2847-2856, 2010.

[17] Y. Li, H. Yuan, A. Von Dem Bussche et al., "Graphene microsheets enter cells through spontaneous membrane penetration at edge asperities and corner sites," Proceedings of the National Academy of Sciences of the United States of America, vol. 110, no. 30, pp. 12295-12300, 2013.

[18] B. Wan, Z.-X. Wang, Q.-Y. Lv et al., "Single-walled carbon nanotubes and graphene oxides induce autophagosome accumulation and lysosome impairment in primarily cultured murine peritoneal macrophages," Toxicology Letters, vol. 221, no. 2, pp. 118-127, 2013.

[19] L. De Marzi, L. Ottaviano, F. Perrozzi et al., "Flake sizedependent cyto and genotoxic evaluation of graphene oxide on in vitro A549, $\mathrm{CaCo} 2$ and vero cell lines," Journal of Biological Regulators and Homeostatic Agents, vol. 28, no. 2, pp. 281-289, 2014.

[20] J. Peng, W. Gao, B. K. Gupta et al., "Graphene quantum dots derived from carbon fibers," Nano Letters, vol. 12, no. 2, pp. 844849, 2012.

[21] B. Z. Ristic, M. M. Milenkovic, I. R. Dakic et al., "Photodynamic antibacterial effect of graphene quantum dots," Biomaterials, vol. 35, no. 15, pp. 4428-4435, 2014.

[22] M. Nurunnabi, Z. Khatun, K. M. Huh et al., "In vivo biodistribution and toxicology of carboxylated graphene quantum dots," ACS Nano, vol. 7, no. 8, pp. 6858-6867, 2013.

[23] S. Stankovich, D. A. Dikin, R. D. Piner et al., "Synthesis of graphene-based nanosheets via chemical reduction of exfoliated graphite oxide," Carbon, vol. 45, no. 7, pp. 1558-1565, 2007.

[24] Z.-S. Wu, W. Ren, L. Gao, B. Liu, C. Jiang, and H.-M. Cheng, "Synthesis of high-quality graphene with a pre-determined number of layers," Carbon, vol. 47, no. 2, pp. 493-499, 2009.

[25] Y. Liu and D. Y. Kim, "Ultraviolet and blue emitting graphene quantum dots synthesized from carbon nano-onions and their comparison for metal ion sensing," Chemical Communications, vol. 51, no. 20, pp. 4176-4179, 2015.

[26] Y. Li, Y. Hu, Y. Zhao et al., "An electrochemical avenue to greenluminescent graphene quantum dots as potential electronacceptors for photovoltaics," Advanced Materials, vol. 23, no. 6, pp. 776-780, 2011.

[27] S. Liu, T. H. Zeng, M. Hofmann et al., "Antibacterial activity of graphite, graphite oxide, graphene oxide, and reduced graphene oxide: membrane and oxidative stress," ACS Nano, vol. 5, no. 9, pp. 6971-6980, 2011.

[28] H.-Q. Chen, D. Gao, B. Wang et al., "Graphene oxide as an anaerobic membrane scaffold for the enhancement of $B$. adolescentis proliferation and antagonistic effects against pathogens E. coli and S. aureus," Nanotechnology, vol. 25, no. 16, Article ID 165101, 2014.

[29] O. N. Ruiz, K. A. S. Fernando, B. Wang et al., "Graphene oxide: a nonspecific enhancer of cellular growth," ACS Nano, vol. 5, no. 10, pp. 8100-8107, 2011.

[30] J. Chen, H. Peng, X. Wang, F. Shao, Z. Yuan, and H. Han, "Graphene oxide exhibits broad-spectrum antimicrobial activity against bacterial phytopathogens and fungal conidia by intertwining and membrane perturbation," Nanoscale, vol. 6, no. 3, pp. 1879-1889, 2014.

[31] J. Li, G. Wang, H. Zhu et al., "Antibacterial activity of largearea monolayer graphene film manipulated by charge transfer," Scientific Reports, vol. 4, article 4359, 2014. 
[32] Y.-W. Wang, A. Cao, Y. Jiang et al., "Superior antibacterial activity of zinc oxide/graphene oxide composites originating from high zinc concentration localized around bacteria," ACS Applied Materials and Interfaces, vol. 6, no. 4, pp. 2791-2798, 2014.

[33] G.-Y. Chen, C.-L. Chen, H.-Y. Tuan et al., "Graphene oxide triggers toll-like receptors/autophagy responses in vitro and inhibits tumor growth in vivo," Advanced Healthcare Materials, vol. 3, no. 9, pp. 1486-1495, 2014.

[34] G.-Y. Chen, C.-L. Meng, K.-C. Lin et al., "Graphene oxide as a chemosensitizer: diverted autophagic flux, enhanced nuclear import, elevated necrosis and improved antitumor effects," Biomaterials, vol. 40, pp. 12-22, 2015.

[35] Y. Wang, S. Wu, X. Zhao, Z. Su, L. Du, and A. Sui, "In vitro toxicity evaluation of graphene oxide on human RPMI 8226 cells," Bio-Medical Materials and Engineering, vol. 24, no. 6, pp. 2007-2013, 2014.

[36] Z. Ding, Z. Zhang, H. Ma, and Y. Chen, "In vitro hemocompatibility and toxic mechanism of graphene oxide on human peripheral blood T Lymphocytes and serum albumin," ACS Applied Materials and Interfaces, vol. 6, no. 22, pp. 19797-19807, 2014. 

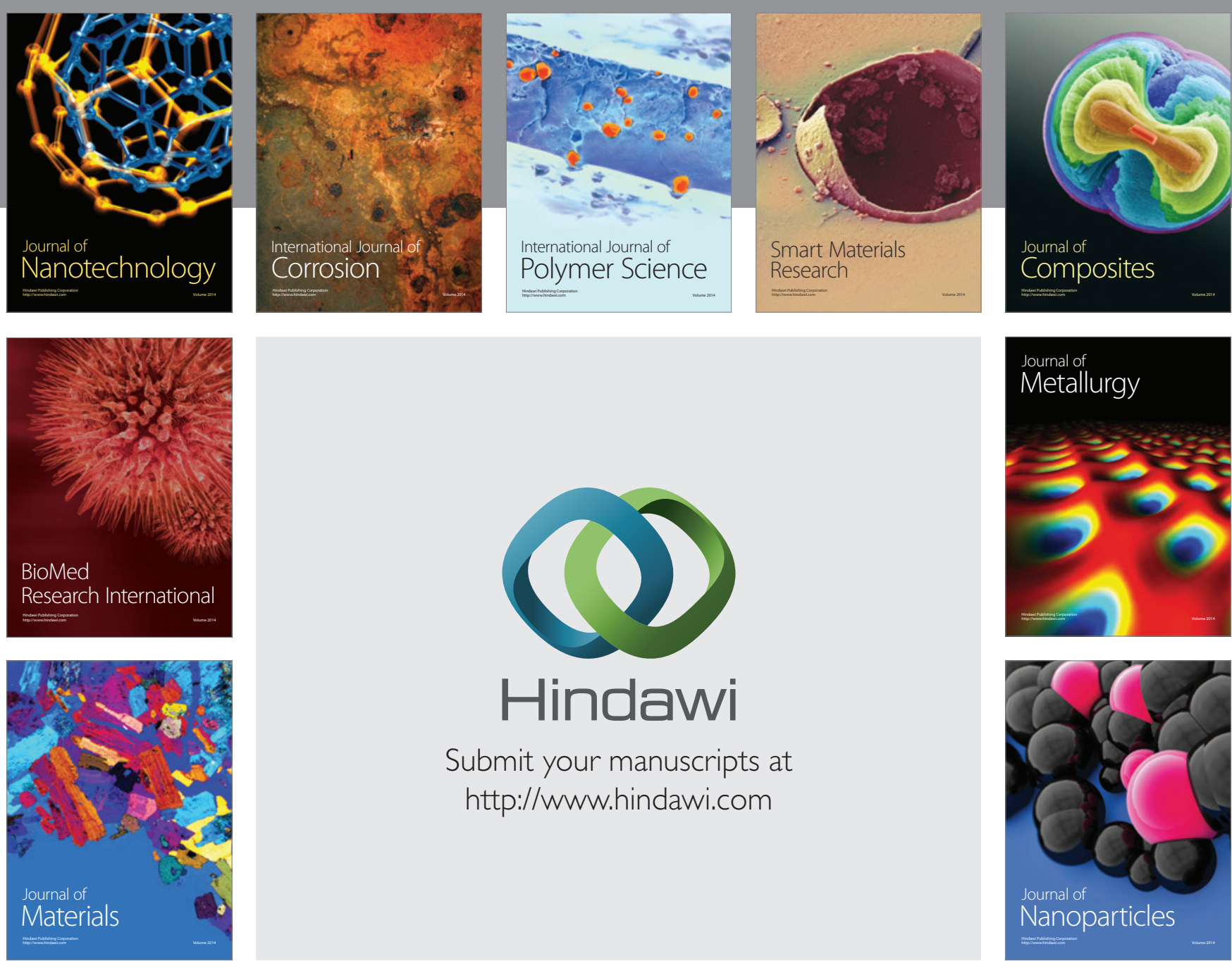

\section{Hindawi}

Submit your manuscripts at

http://www.hindawi.com

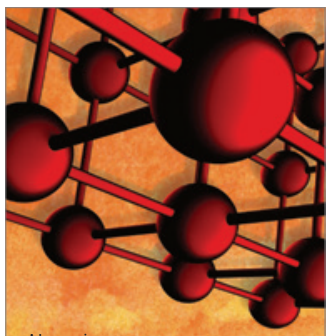

Materials Science and Engineering
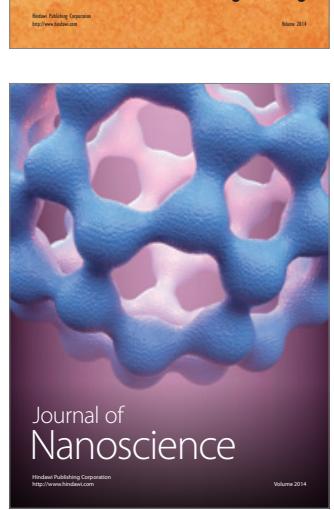
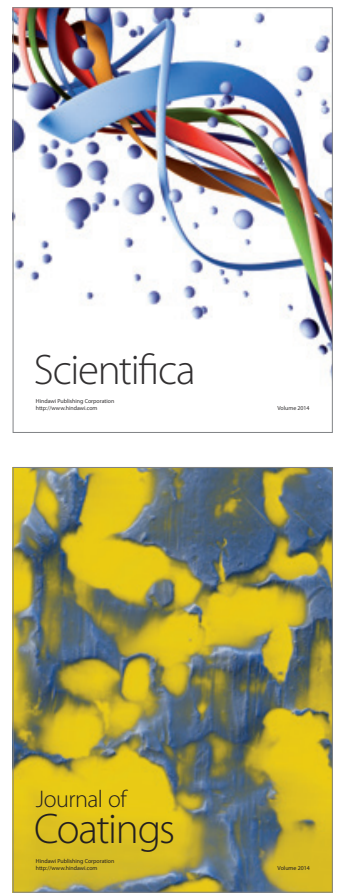
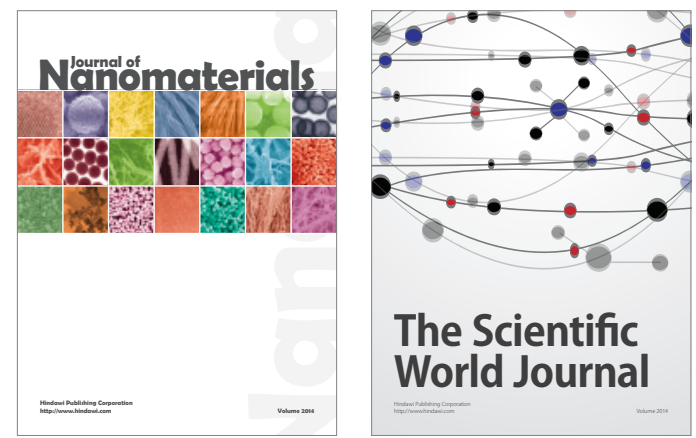

The Scientific World Journal
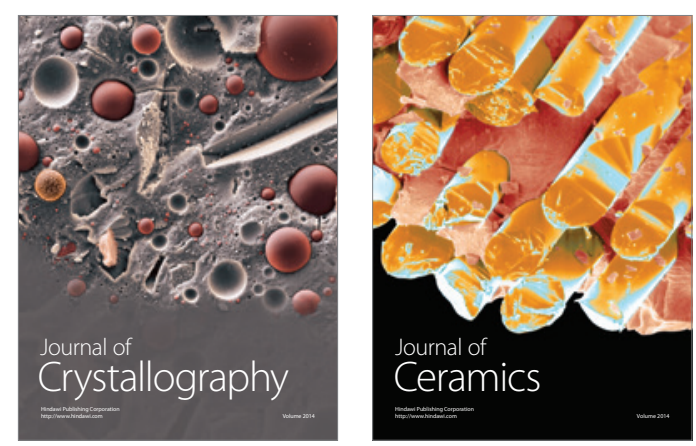
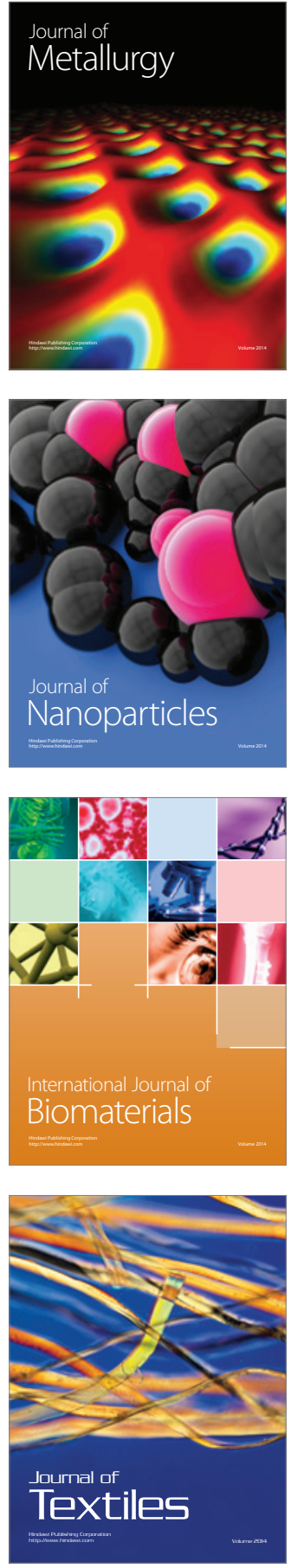